\title{
La transgression : de l'émancipation à la progression
}

\section{Marie-Noëlle COCTON, Hélène FAVREAU, Sophie ROCH-VEIRAS}

Le dossier qui va suivre fait suite à un colloque en langues, cultures et apprentissages organisé du 30 septembre au 1 ${ }^{\text {er }}$ octobre 2013 à l'Université Catholique de l'Ouest par le laboratoire CoDiRe (Construction Discursive des Représentations linguistiques et culturelles) de l'Université de Nantes et l'équipe associée du LICIA (Langages, Interactions Culturelles, Identités et Apprentissages) à l'UCO.

Le terme de transgression signifie, comme son nom l'indique en latin, «passer de l'autre côté », «traverser ». Attesté dès le vingtième siècle dans la langue française, il réfère au non-respect de règles et d'ordres. Incitant à passer «au-delà des frontières », il pose l'existence de normes préétablies, cette existence de normes faisant apparaitre les transgressions. Celui qui transgresse est immanquablement un être subversif qui rejette la règle ou la norme elle-même. Avec le siècle des Lumières, ce concept est toujours lié au fait de «passer outre». Néanmoins, aussi bien dans le domaine social, philosophique que politique, les normes jusque-là en vigueur peuvent désormais être contestées voire transgressées. En s'en emparant, au tout début du XXe siècle, la géologie, en opposant la régression à la transgression finira par donner à ce terme un sens mélioratif.

Dès lors, elle prend «le sens d'une progression ou d'une régression selon le statut reconnu au monde qui est garanti par le respect de la règle bafouée »(Folscheid). Même si la transgression implique la notion de limite, "tout dépassement d'une limite n'est [de fait] pas pour autant une transgression » (Folscheid) et la limite est bien ce qui peut donner forme à la norme. Dans toute institution où l'on apprend une langue et une culture, de l'école à l'université, nous sommes soumis à des normes. La langue, comme système linguistique, répond elle aussi à des exigences. Du fait même de la présence d'un Autre, nous sommes contraints à des limites ; l'Autre, lui-même confronté à un limitant, se heurte lui aussi à des limites.

Si l'on envisage ainsi la transgression non plus comme le simple fait de contrevenir à une règle, mais d'abord, comme une co-construction entre une personne et des situations, et plutôt comme un mouvement en avant, et pourquoi pas, comme «un chemin de traverse, avec un certain goût de l'aventure» où nous pourrions «retrouver le goût de la clandestinité »(Meirieu), nous touchons une perspective qui nous permet d'aborder ce phénomène également comme une invitation à dépasser, voire même à outrepasser. 
C'est cette dimension constructive de la notion de transgression linguistique et culturelle que nous souhaitons privilégier ici, pour tâcher de lui rendre la richesse de sa valeur progressive, celle qui peut nous faire aller de l'avant et pour cela compléter le concept de limite. Selon cette optique, on s'interrogera alors sur un nouvel espace à trouver ou à créer dans une tension entre transgression de règles et désir de s'émanciper.

Devant l'ampleur et la complexité de la question, trois axes thématiques ont été privilégiés :

- Le premier est de l'ordre des sciences du langage. La réflexion s'articulera autour de notions déjà abordées comme celles de l'écart, du dépassement d'une limite, de la transgression d'une règle mais aussi de variante et de variation et de leurs conséquences sur le plan de la langue, du discours et de la communication. Berthille Pallaud envisage le caractère évolutif de la transgression en prenant appui sur les spécificités de la langue orale (disfluences) alors que Tomonori Okubo s'intéresse aux énoncés ironiques. Hélène Favreau, quant à elle, analyse les influences réciproques du discours publicitaire sur la langue. En prenant l'exemple du gérondif non coréférentiel, Jun-Ya Watanabe fait ressortir son emploi souvent qualifié d'anomalie grammaticale. Magali Italia s'interroge sur le français parlé au Gabon et ses transformations.

- La deuxième perspective relève de la didactique des langues-cultures et plus largement des sciences de l'éducation et de la psychologie. Enseignants comme apprenants, nous sommes tous régis par les lois d'une école, d'un institut, d'une université ou d'un centre de formation, eux-mêmes soumis, dans une certaine mesure, au Cadre européen commun de référence pour les langues (CECR). A travers l'exemple des enfants plurilingues, Marie Berchoud montre comment la transgression d'une norme ou une autre peut être une prise de conscience et un révélateur. Laura Nicolas interroge le rôle potentiellement transgressif des interventions de l'enseignant lors des interactions avec les apprenants tandis que Valérie Soubre considère l'évaluation formative comme source d'émancipation. Sur un plan plus concret, Marine Abraham, Syrine Diaz-Daoussi, Marie Andre-Milesi et Alex Cormanski s'inscrivent dans une réflexion plus pragmatique dans l'utilisation des affiches publicitaires, des proverbes, de la poésie et du corps dans la classe de langue. Pour Assmaâ Afnakar, l'introduction des TICE, au Maroc, semble être un moyen de transgression des contraintes institutionnelles et de celles liées à l'apprentissage. Au 
Liban, Isabelle Grappe nous montre que le recours à l'arabe courant constitue une transgression positive.

- Quelle place accorder aux formes de transgression ? Transgresser, est-ce obéir ou innover? La troisième perspective, dans le numéro de Voix plurielles à paraitre en décembre 2015, se placera d'un point de vue anthropologique. Une norme n'est légitime que si elle est acceptée par un groupe social. Dès lors, ce qui est tenu pour une conduite conforme dépend de la validité que lui accordent ceux qui l'acceptent. Lorsque l'on est étranger, l'acceptation de la norme «de l'autre » ne va pas de soi. Pourtant, comme l'a souligné Martine Abdallah Pretceille, de l'école à l'université, enseignants ou apprenants, «chacun apporte avec lui ses propres capitaux culturels et ses habitus singuliers, ses héritages et ce qui le définit comme individu unique ». Pour Anne-Charlotte Taillandier, qui étudie la population de jeunes handicapés, la transgression passe par une volonté de dépasser le handicap, pour les personnes handicapées, et pour ces dernières de s'impliquer dans leurs apprentissages. Pour Zaïha Zeroulou, l'émigration déclenche une dynamique de transgressions plurielles passant par le refus, pour l'immigré, de sa condition. Dans le monde de la littérature aussi, la transgression est souvent suggérée par une échappée de normes, comme le suggèrent Belfar Boubaaya Naciera dans ses comparaisons entre Assia Djebar et Charlotte Brontë ; et Audrey Ogès, entre Déwé Görödé et Chantal Spitz.

\section{Références}

Abdallah-Pretceille, Martine et Louis Porcher. Éducation et communication interculturelle. 2e éd. Paris : PUF, 2001.

Brizard Philippe et Dominique Folscheid (collectif). La transgression. Paris : FrançoisXavier de Guibert, 2005.

Meirieu, Philippe. «Apprentissage et transgression». Texte de conférence donnée dans le cadre des entretiens Nathan, 2003.

Pallaud, Berthille. «La transgression et la variation ». Marges linguistiques 8 (2004) : 76-87. [http://www.marges-linguistiques.com] (dernière consultation le 15/09/12). 